\title{
Synthesis and Evaluation of Tumor Cell Growth Inhibition of Methyl 3-Amino-6-[(hetero)arylethynyl]thieno[3,2-b]pyridine-2-carboxylates. Structure-Activity Relationships, Effects on the Cell Cycle and Apoptosis
}

\author{
Maria-João R. P. Queiroz, ${ }^{, a}$ Ricardo C. Calhelha, ${ }^{a}$ Luís A. Vale-Silva, ${ }^{\text {E }}$ Eugénia Pinto, ${ }^{\text {b }}$ \\ Gabriela M. Almeida, ${ }^{\mathrm{c}}$ M. Helena Vasconcelos ${ }^{\mathrm{b}, \mathrm{c}}$ \\ ${ }^{a}$ Centro de Química, Universidade do Minho, Campus de Gualtar, 4710-057 Braga, Portugal \\ ${ }^{b}$ Serviço de Microbiologia / Centro de Química Medicinal da Universidade do Porto (CEQUIMED-UP), Faculdade \\ de Farmácia da Universidade do Porto, Rua Aníbal Cunha 164, 4050-047 Porto, Portugal \\ ${ }^{c}$ Cancer Biology Group, IPATIMUP - Institute of Molecular Pathology and Immunology of the University of Porto, \\ Rua Dr. Roberto Frias, 4200-465 Porto, Portugal.
}

\author{
* Corresponding author \\ Maria-João R. P. Queiroz \\ Centro de Química, Universidade do Minho, Campus de Gualtar, 4710-057 Braga, Portugal \\ Tel: +351253604378; Fax: +351253604382 \\ e-mail: mjrpq@quimica.uminho.pt
}

\begin{abstract}
The methyl 3-amino-6-bromothieno[3,2-b]pyridine-2-carboxylate, recently reported by some of us, was reacted in Sonogashira couplings with several (hetero)arylacetylenes. The growth inhibitory activity of the novel methyl 3-amino-6[(hetero)arylethynyl]thieno[3,2-b]pyridine-2-carboxylates obtained was evaluated on three human tumor cell lines (MCF-7, NCI-H460, A375-C5). The para-methoxyphenyl and the ortho and para-aminophenyl derivatives were the most promising compounds, and their effects were further studied regarding alterations in the normal cell cycle distribution and induction of apoptosis in the NCI-H460 cell line. All three compounds altered cell cycle distribution and the ortho-aminophenyl derivative was further shown to induce apoptosis in the same cell line.
\end{abstract}

Keywords: Thieno[3,2-b]pyridines, Sonogashira coupling, Antitumoral activity, Cell Cycle, Apoptosis

\section{Introduction}

Recently several thieno[3,2- $b]$ pyridines have shown important biological activities, namely antitumor and antiangiogenesis or dual activity, essentially by acting as inhibitors of tyrosine kinase receptors I [1], II [2] III [3], or nonreceptors (IV) [4] which have been implicated in the growth and progression of various human cancers and, therefore, have been crucial in the development of anticancer therapies. 
<smiles></smiles>

I<smiles>CCn1cnc(-c2cc3nccc(Oc4ccc(NC(=O)N5CCN(c6ccc(F)cc6)C5=O)cc4F)c3s2)c1</smiles>

III<smiles>O=C(Cc1ccccc1)NC(=S)Nc1ccc(Oc2ccnc3cc(C(F)(F)F)sc23)c(F)c1</smiles>

II<smiles>COc1cc(Nc2c(C#N)cnc3cc([Al])sc23)c(Cl)cc1Cl</smiles>

IV

As we have recently prepared the methyl 3-amino-6-bromothieno[3,2- $b$ ]pyridine-2carboxylate [5] it was decided to use it in the $\mathrm{Pd} / \mathrm{Cu}$-catalysed Sonogashira coupling [6] with several terminal (hetero)arylacetylenes in the synthesis of novel methyl 3-amino-6[(hetero)arylethynyl]thieno[3,2- $b]$ pyridine-2-carboxylates. Their potential as antitumor agents was evaluated through the in vitro growth inhibitory activity on three selected human tumor cell lines, MCF-7, NCI-H460, and A375-C5 and some structure-activity relationships (SARs) were established.

For the most promising compounds the effects on the cell cycle distribution and induction of apoptosis were studied, in order to investigate their possible mechanisms of action.

\section{Results and discussion}

\subsection{Synthesis}

The 6-bromothieno[3,2-b]pyridine 1 [5] was used as the thieno[3,2-b]pyridine component in the $\mathrm{Pd} / \mathrm{Cu}$ catalyzed Sonogashira reaction [5-7] with several (hetero)arylacetylenes to obtain the novel methyl 3-amino-6[(hetero)arylethynyl]thieno[3,2-b]pyridine-2-carboxylates $\mathbf{2 a - m}$ in high to excellent yields using the conditions depicted in Scheme 1, already used by us in the synthesis of arylethynyl benzo[ $b]$ thiophene derivatives [7]. 


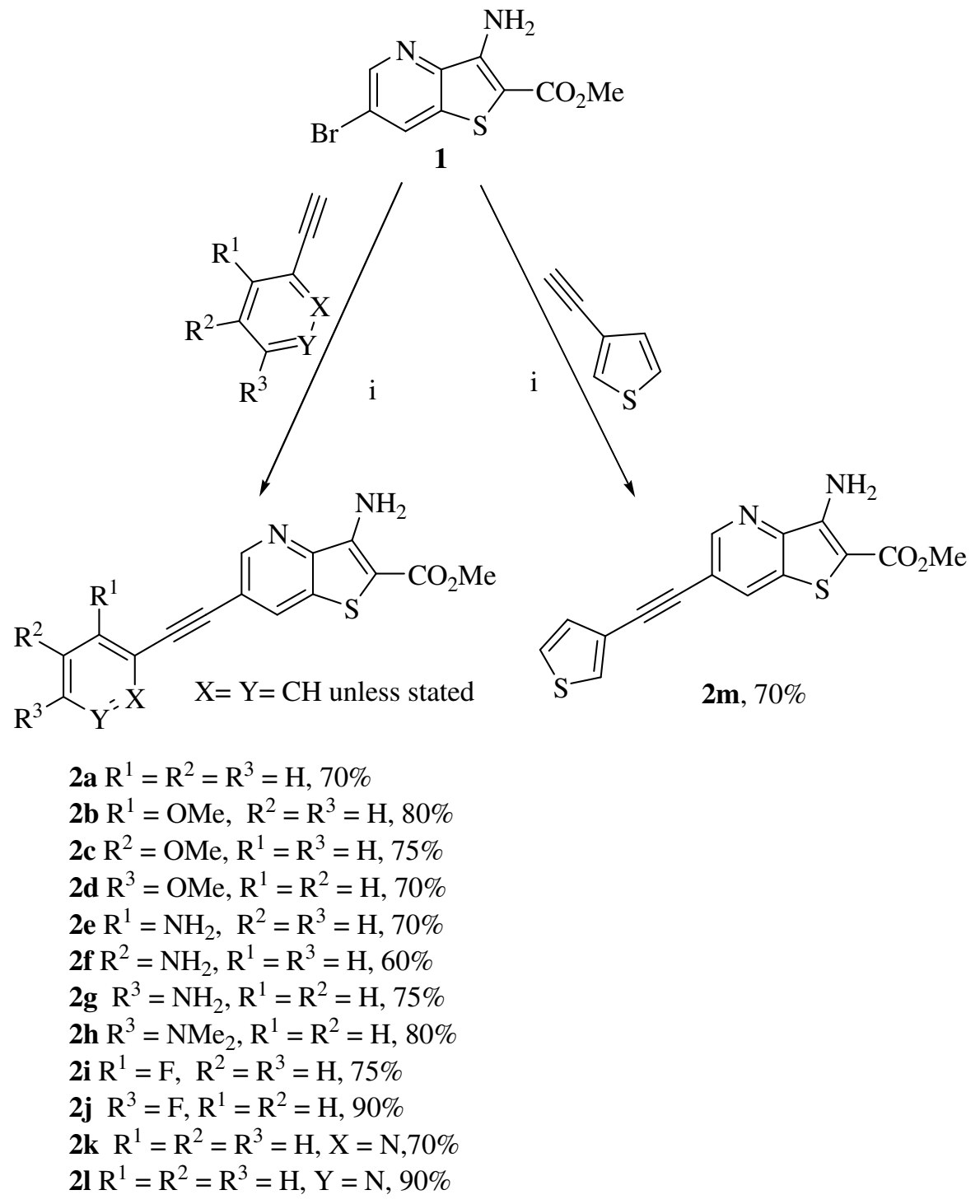

Scheme 1. Synthesis of compounds 2a-2m. Conditions: $\mathrm{PdCl}_{2}\left(\mathrm{PPh}_{3}\right)_{2}(5 \mathrm{~mol} \%), \mathrm{CuI}(3 \mathrm{~mol} \%), \mathrm{NEt}_{3}(3$ equiv.), DMF, $1.5 \mathrm{~h}, 100{ }^{\circ} \mathrm{C}$.

\subsection{Growth inhibition of human tumor cell lines}

The in vitro growth inhibitory activity of all the compounds prepared was evaluated using three human tumor cell lines, representing different tumor types, namely breast adenocarcinoma (MCF-7), melanoma (A375-C5), and non-small cell lung cancer (NCIH460), after a continuous exposure of $48 \mathrm{~h}$. The results are summarized in Table 1. 
Table 1- Growth inhibitory activity of the 6-(hetero)arylethynylthieno[3,2-b]pyridines 2 on the three human tumor cell lines in study.

\begin{tabular}{cccc}
\hline \multirow{2}{*}{ Compounds } & \multicolumn{3}{c}{$\mathbf{G I}_{\mathbf{5 0}}(\boldsymbol{\mu M})^{\mathbf{a}}$} \\
\cline { 2 - 4 } & $\mathbf{M C F}-7$ & $\mathbf{A 3 7 5}^{\mathbf{C} 5}$ & $\mathbf{N C I - H 4 6 0}$ \\
\hline $\mathbf{2 a}$ & $>150.0$ & $>150.0$ & $>150.0$ \\
\hline $\mathbf{2 b}$ & $>47.3^{\mathrm{b}}$ & $>47.3^{\mathrm{b}}$ & $>47.3^{\mathrm{b}}$ \\
\hline $\mathbf{2 c}$ & $33.4 \pm 4.7$ & $>75.0^{\mathrm{b}}$ & $49.0 \pm 4.7$ \\
\hline $\mathbf{2 d}$ & $0.46 \pm 0.05$ & $>37.5^{\mathrm{b}}$ & $0.32 \pm 0.05$ \\
\hline $\mathbf{2}$ & $4.1 \pm 0.7$ & $3.1 \pm 2.1$ & $5.7 \pm 3.0$ \\
\hline $\mathbf{2 f}$ & $10.8 \pm 1.2$ & $11.6 \pm 0.6$ & $11.5 \pm 0.5$ \\
\hline $\mathbf{2 g}$ & $2.6 \pm 1.4$ & $4.8 \pm 0.4$ & $8.9 \pm 0.9$ \\
\hline $\mathbf{2 h}$ & $20.6 \pm 1.1$ & $17.7 \pm 0.1$ & $16.6 \pm 1.1$ \\
\hline $\mathbf{2 i}$ & $>150$ & $>150$ & $>150$ \\
\hline $\mathbf{2 j}$ & $29.0 \pm 1.2$ & $48.4 \pm 1.6$ & $24.0 \pm 2.0$ \\
\hline $\mathbf{2 k}$ & $37.4 \pm 4.1$ & $13.9 \pm 1.9$ & $34.9 \pm 5.4$ \\
\hline $\mathbf{2}$ & $>150$ & $>150$ & $>150$ \\
\hline $\mathbf{2 m}$ & $27.7 \pm 2.7$ & $29.6 \pm 0.2$ & $24.8 \pm 0.5$
\end{tabular}

${ }^{\mathrm{a}}$ The lowest concentrations causing $50 \%$ of cell growth inhibition $\left(\mathrm{GI}_{50}\right)$ after a continuous exposure of $48 \mathrm{~h}$, expressed as means \pm SEM of three independent experiments performed in duplicate. ${ }^{\mathrm{b}}$ Due to the low solubility of the compound in DMSO the maximum concentration tested was lower than $150 \mu \mathrm{M}$. Doxorubicin was used as positive control (GI50: MCF-7 = 43.3 $\pm 2.6 \mathrm{nM}$; A375-C5 = 130.2 $\pm 10.1 \mathrm{nM} ; \mathrm{NCI}-\mathrm{H} 460=35.6 \pm 1.6 \mathrm{nM})$. 
From the analysis of Table 1 it can be concluded that the $p$-methoxylated compound $\mathbf{2 d}$ presents the lowest $\mathrm{GI}_{50}$ values (below $1.0 \mu \mathrm{M}$ ), with marked selectivity for MCF-7 and NCI-H460 cell lines. The aminated compounds $\mathbf{2 e - 2 g}$ were also effective at inhibiting cell growth, with $\mathrm{GI}_{50}$ values between 2.6-11.6 $\mu \mathrm{M}$ for the three cell lines, the best being the $o$ - and $p$-aminophenyl compounds $2 \mathbf{e}$ and $\mathbf{2 g}$.

The methoxylated compounds $\mathbf{2 b}$ and $\mathbf{2 c}$ were not very potent at inhibiting cell growth. They are not very soluble, nevertheless for compound $\mathbf{2 c}$ it was possible to determine the $\mathrm{GI}_{50}$ values for MCF-7 and NCI-H460 which were between 33.4 and $49.0 \mu \mathrm{M}$. The tendency for selective activity excluding the melanoma cell line (A375-C5), which is clear for compound $\mathbf{2 d}$, is already detectable in $\mathbf{2 c}$. The $p$-dimethylamino derivative $\mathbf{2 h}$ presents higher $\mathrm{GI}_{50}$ values than the free amino compounds. Among the fluorinated compounds $\mathbf{2} \mathbf{i}$ and $\mathbf{2} \mathbf{j}$ only the latter shows moderate $\mathrm{GI}_{50}$ values $(24.0-48.4 \mu \mathrm{M})$. Considering the fact that the unsubstituted phenylacetylene derivative 2a did not show any activity at the highest tested concentration, the cell growth inhibitory activity presented by the compounds discussed above appears to be closely dependent on the substitution pattern.

The pyridine derivative $\mathbf{2 k}$ shows some selectivity against A375-C5 cell line, with a $\mathrm{GI}_{50}$ value of $13.9 \mu \mathrm{M}$. The position of the $\mathrm{C}-\mathrm{C}$ bond in the pyridine ring appears to be determinant for the cell growth inhibitory activity, since compound $\mathbf{2 l}$ was not active at the highest concentration tested.

The thiophene derivative $\mathbf{2} \mathbf{m}$ presents moderate growth inhibitory activity against the three cell lines in study $\left(\mathrm{GI}_{50}\right.$ values between 24.8-29.6 $\left.\mu \mathrm{M}\right)$.

\subsection{Cell cycle analysis and detection of apoptosis}

The most active compounds were chosen to be further investigated regarding their mechanism of action. The NCI-H460 cell line was incubated with the $\mathrm{GI}_{50}$ concentrations of compounds $\mathbf{2 d}, \mathbf{2 e}$ and $\mathbf{2 g}$ for $48 \mathrm{~h}$ and their effects on the normal cell cycle distribution and induction of apoptosis analyzed. The results show that all three compounds interfere with the normal cell cycle distribution of this cell line. Compound 2d caused a small increase in the percentage of cells in G0/G1 and a reduction in the percentage of cells in the S-phase of the cell cycle (Table 2). A similar but more marked effect was observed upon incubation with compound $\mathbf{2 g}$. Compound $\mathbf{2 e}$ induced an increase in the percentage of cells in $\mathrm{G} 2 / \mathrm{M}$ phases of the cell cycle accompanied by a 
decrease of cells in S-phase (Table 2). From these results it appears that compounds $2 \mathbf{d}$ and $\mathbf{2 g}$ induce a cell cycle arrest on phases G0/G1, whereas compound $\mathbf{2 e}$ appears to cause a G2/M cell cycle arrest. These compounds were also shown to induce apoptosis, as determined by the annexin V-FICT/PI flow cytometry assay, particularly compound 2e (Table 2). This observation was confirmed by the presence of a sub-G1 peak in the cell cycle profile analysis (indicative of DNA degradation, characteristic of apoptosis) upon incubation with compound $\mathbf{2 e}$ (data not shown).

Table2. Cell cycle analysis (\% of cells in phases G0/G1, S, G2/M) and $\%$ of NCI-H460 cells undergoing apoptosis after a $48 \mathrm{~h}$ treatment with compounds $\mathbf{2 d}, \mathbf{2 e}$ and $\mathbf{2 g}$ at their $\mathrm{GI}_{50}$ concentrations.

\begin{tabular}{ccccc}
\hline & $\begin{array}{c}\text { G0/G1 } \\
(\text { cells \% })\end{array}$ & $\begin{array}{c}\text { S } \\
(\text { cells \%) }\end{array}$ & $\begin{array}{c}\text { G2/M } \\
(\text { cells \%) }\end{array}$ & $\begin{array}{c}\text { Apoptosis } \\
(\text { cells \%) }\end{array}$ \\
\hline Control & $57.1 \pm 1.6$ & $28.2 \pm 1.4$ & $14.7 \pm 0.9$ & $11.7 \pm 1.6$ \\
DMSO & $55.1 \pm 1.0$ & $28.8 \pm 1.2$ & $16.1 \pm 0.9$ & $12.2 \pm 1.2$ \\
$\mathbf{2 d}$ & $59.7 \pm 3.2$ & $22.0 \pm 1.7$ & $18.3 \pm 1.9$ & $18.7 \pm 2.8$ \\
$\mathbf{2 e}$ & $54.7 \pm 2.7$ & $23.1 \pm 2.8$ & $24.6 \pm 2.4$ & $25.3 \pm 1.8^{\mathrm{a}}$ \\
$\mathbf{2 g}$ & $68.0 \pm 0.7$ & $18.6 \pm 0.8$ & $13.4 \pm 0.6$ & $17.0 \pm 2.1$
\end{tabular}

Untreated cells were used as the control and a solvent control (DMSO) was also included. Results are the mean \pm standard error of three to six independent experiments performed in duplicate.

${ }^{a}$ Apoptosis induction by compound $\mathbf{2 e}$ was statistically significantly when compared to the control $(P<0.05)$.

According to these findings, the compounds are likely to present different mechanisms of action. The effect of compound $\mathbf{2} \mathbf{e}$ in cell growth inhibition seems to be due to the induction of both apoptosis and cell cycle arrest in the G2/M phase (Table2). Compounds $\mathbf{2 d}$ and $\mathbf{2 g}$ did not cause such a marked induction of apoptosis (Table 2) and thus their growth inhibitory effects seem to be mainly due to cell cycle related events.

\section{Conclusion}

Novel methyl 3-amino-6-[(hetero)arylethynyl]thieno[3,2-b]pyridine-2-carboxylates were synthesized and tested for their in vitro growth inhibitory activity on three human tumor cell lines. All compounds were fully soluble at the $\mathrm{GI}_{50}$ concentration. Overall, the para-methoxyphenyl and the ortho and para-aminophenyl derivatives are the most 
promising compounds, presenting very low $\mathrm{GI}_{50}$ values and therefore their effects on cell cycle distribution and induction of apoptosis were further investigated in the NCIH460 cell line. Compounds $\mathbf{2 d}$, 2e and $\mathbf{2 g}$ had an effect on cell cycle distribution, with a G0/G1 arrest being detected for the para-methoxyphenyl and the para-aminophenyl derivatives and a G2/M arrest for the ortho-aminophenyl derivative. Additionally, the ortho-aminophenyl compound was found to significantly induce apoptosis at the $\mathrm{GI}_{50}$ concentration in the tested cell line. Hence, at least two mechanisms of action appear to be involved in the activity of this compound, cell cycle related events and induction of apoptosis. The obtained data suggest that the results are dependent on the compound substitution pattern, nevertheless it is important to bear in mind that the physical properties of the compounds, particularly cell permeability, may be influencing their relative activity.

\section{Experimental}

\subsection{Synthesis}

Melting points $\left({ }^{\circ} \mathrm{C}\right)$ were determined in a SMP3 Stuart apparatus and are uncorrected. ${ }^{1} \mathrm{H}$ and ${ }^{13} \mathrm{C}$ NMR spectra were recorded on a Varian Unity Plus at 300 and $75.4 \mathrm{MHz}$, and on a Bruker Avance II at 400 and 100.6 MHz, respectively. Heteronuclear correlations, ${ }^{1} \mathrm{H}-{ }^{13} \mathrm{C}$, HMQC or HMSQ and HMBC were performed to attribute some signals. MS (EI) and HRMS data were recorded by the mass spectrometry service of the University of Vigo, Spain. Elemental analysis was performed on a LECO CHNS 932 elemental analyser. The reactions were monitored by thin layer chromathography (TLC). Dry flash was performed on Macherey-Nagel silica gel 150-230 mesh. Petroleum ether refers to the boiling range 40-60 ${ }^{\circ} \mathrm{C}$. Ether refers to diethylether.

\subsubsection{General procedure for the synthesis of compounds $2 \mathrm{a}-2 \mathrm{~m}$ :}

Compound 1 (150 mg, $0.540 \mathrm{mmol}$ ), the (hetero)arylacetylene (1.1 equiv.), $\mathrm{PdCl}_{2}\left(\mathrm{PPh}_{3}\right)_{2}(5 \mathrm{~mol} \%), \mathrm{CuI}$ ( 3 mol\%), and $\mathrm{NEt}_{3}$ (3 equiv.) were added to dry DMF (2-3 mL) into a dry Schlenk tube, under argon, and the mixture was heated with stirring at $100{ }^{\circ} \mathrm{C}$ for $1.5 \mathrm{~h}$. After cooling, water $(5 \mathrm{~mL})$ and ethyl acetate $(5 \mathrm{~mL})$ were added and the phases were separated. The aqueous phase was then extracted with additional ethyl acetate $(3 \times 5 \mathrm{~mL})$. The organic phases were collected, dried $\left(\mathrm{MgSO}_{4}\right)$, filtered, and, finally, removal of the solvent under reduced pressure gave a solid which was submitted to column chromatography using $50 \%$ ether/ petroleum ether as eluent. The solid obtained was washed with petroleum ether and fully characterized.

4.1.1.1. Methyl 3-amino-6-(2-phenylethynyl)thieno[3,2-b]pyridine-2-carboxylate (2a): Yellow solid (117 mg, 70\%), m.p. $175-177^{\circ} \mathrm{C} .{ }^{1} \mathrm{H}$ NMR $\left(\mathrm{CDCl}_{3}, 400 \mathrm{MHz}\right) \delta 3.93(3 \mathrm{H}, \mathrm{s}, \mathrm{OMe}), 6.24\left(2 \mathrm{H}\right.$, br s, $\left.\mathrm{NH}_{2}\right)$, 7.38-7.42 (3H, m, Ar-H), 7.56-7.60 (2H, m, Ar-H), $8.18(1 \mathrm{H}, \mathrm{d}, J=2 \mathrm{~Hz}, \mathrm{Ar}-\mathrm{H}), 8.73(1 \mathrm{H}, \mathrm{d}, J=2 \mathrm{~Hz}$, $\mathrm{Ar}-\mathrm{H}) \mathrm{ppm} .{ }^{13} \mathrm{C} \mathrm{NMR}\left(\mathrm{CDCl}_{3}, 100.6 \mathrm{MHz}\right) \delta 51.75(\mathrm{OMe}), 86.07(\mathrm{C}), 93.88(\mathrm{C}), 100.84(2-\mathrm{C})$, 119.11(2×CH), $122.29\left(1^{\prime}-\mathrm{C}\right), 128.50(2 \times \mathrm{CH}), 129.02(\mathrm{C}), 131.74(\mathrm{C}), 133.49(\mathrm{CH}), 133.72(\mathrm{C}), 144.97$ (3a-C), $147.30(\mathrm{C}), 148.73(\mathrm{CH}), 165.17(\mathrm{C}=\mathrm{O}) \mathrm{ppm}$. MS (EI) m/z $308\left(\mathrm{M}^{+}, 100\right), 276(66)$. HRMS M calcd. for $\mathrm{C}_{17} \mathrm{H}_{12} \mathrm{~N}_{2} \mathrm{O}_{2} \mathrm{~S} 308.0619$; found 308.0624 .

4.1.1.2. Methyl 3-amino-6-[2-(2-methoxyphenyl)ethynyl]thieno[3,2-b]pyridine-2-carboxylate (2b): Yellow solid (146 mg, 80\%), m.p. 193-195 ${ }^{\circ} \mathrm{C} .{ }^{1} \mathrm{H}$ NMR $\left(\mathrm{CDCl}_{3}, 400 \mathrm{MHz}\right) \delta 3.92(3 \mathrm{H}, \mathrm{s}, \mathrm{OMe}), 3.95$ $(3 \mathrm{H}, \mathrm{s}, \mathrm{OMe}), 6.22\left(2 \mathrm{H}\right.$ br s, $\left.\mathrm{NH}_{2}\right), 6.93-6.98(2 \mathrm{H}, \mathrm{m}, \mathrm{Ar}-\mathrm{H}), 7.34-7.39(1 \mathrm{H}, \mathrm{m}, \mathrm{Ar}-\mathrm{H}), 7.53(1 \mathrm{H}, \mathrm{dd}, J=$ 7.6 and $2 \mathrm{~Hz}, \mathrm{Ar}-\mathrm{H}), 8.20(1 \mathrm{H}, \mathrm{d}, J=2 \mathrm{~Hz}, \mathrm{Ar}-\mathrm{H}), 8.75(1 \mathrm{H}, \mathrm{d}, J=2 \mathrm{~Hz}, \mathrm{Ar}-\mathrm{H}) \mathrm{ppm} .{ }^{13} \mathrm{C} \mathrm{NMR}\left(\mathrm{CDCl}_{3}\right.$, 
100.6 MHz) $\delta 51.71(\mathrm{OMe}), 55.83(\mathrm{OMe}), 90.06(\mathrm{C}), 90.45(\mathrm{C}), 100.68(2-\mathrm{C}), 110.74(\mathrm{CH}), 111.52(\mathrm{C})$, $119.50(\mathrm{C}), 120.57(\mathrm{CH}), 130.57(\mathrm{CH}), 133.38(\mathrm{CH}), 133.65(\mathrm{CH}), 133.67(\mathrm{C}), 144.89(\mathrm{C}), 147.38(\mathrm{C})$, $148.89(\mathrm{CH}), 160.16(\mathrm{C}), 165.21(\mathrm{C}=\mathrm{O}) \mathrm{ppm}$. MS (EI) m/z $338\left(\mathrm{M}^{+}, 100\right), 306(39) . \mathrm{HRMS} \mathrm{M}^{+}$calcd. for $\mathrm{C}_{18} \mathrm{H}_{14} \mathrm{~N}_{2} \mathrm{O}_{3} \mathrm{~S} 338.0725$; found 338.0727.

41.1.3. Methyl 3-Amino-6-[2-(3-methoxyphenyl)ethynyl]thieno[3,2-b]piridine-2-carboxylate (2c): Yellow solid (137 mg, 75\%), m.p. 177-179 ${ }^{\circ} \mathrm{C} .{ }^{1} \mathrm{H}$ NMR $\left(\mathrm{CDCl}_{3}, 400 \mathrm{MHz}\right) \delta 3.85$ (3H, s, OMe), 3.93 $(3 \mathrm{H}, \mathrm{s}, \mathrm{OMe}), 6.23\left(2 \mathrm{H}\right.$, br s, $\left.\mathrm{NH}_{2}\right), 6.94-6.97(1 \mathrm{H}, \mathrm{m}, \mathrm{Ar}-\mathrm{H}), 7.09-7.11(1 \mathrm{H}, \mathrm{m}, \mathrm{Ar}-\mathrm{H}), 7.16-7.19(1 \mathrm{H}, \mathrm{m}$, Ar-H), 7.30 (1H, app. t, $\left.J=8 \mathrm{~Hz}, 5^{\prime}-\mathrm{H}\right), 8.19(1 \mathrm{H}, \mathrm{d}, J=1.6 \mathrm{~Hz}, \mathrm{Ar}-\mathrm{H}), 8.73(1 \mathrm{H}, \mathrm{d}, J=1.6 \mathrm{~Hz}, \mathrm{Ar}-\mathrm{H})$ ppm. ${ }^{13} \mathrm{C} \mathrm{NMR}\left(\mathrm{CDCl}_{3}, 100.6 \mathrm{MHz}\right) \delta 51.74(\mathrm{OMe}), 55.33(\mathrm{OMe}), 85.89(\mathrm{C}), 93.78(\mathrm{C}), 100.84(2-\mathrm{C})$, $115.65(\mathrm{CH}), 116.48(\mathrm{CH}), 119.03(\mathrm{C}), 123.26(\mathrm{C}), 124.29(\mathrm{CH}), 129.58\left(5^{\prime}-\mathrm{CH}\right), 133.49(\mathrm{CH}), 133.69$ (C), $145.05(\mathrm{C}), 147.32(\mathrm{C}), 148.78(\mathrm{CH}), 159.42(\mathrm{C}), 165.18(\mathrm{C}=\mathrm{O}) \mathrm{ppm} . \mathrm{MS}(\mathrm{EI}) \mathrm{m} / \mathrm{z} 338\left(\mathrm{M}^{+}, 100\right)$, 306 (53). HRMS M${ }^{+}$calcd. for $\mathrm{C}_{18} \mathrm{H}_{14} \mathrm{~N}_{2} \mathrm{O}_{3} \mathrm{~S} 338.0725$; found 338.0723.

4.1.1.4. Methyl 3-amino-6-[2-(4-methoxyphenyl)ethynyl]thieno[3,2-b]pyridine-2-carboxylate (2d): yellow solid (130 mg, $70 \%$ ), m.p. $190-192{ }^{\circ} \mathrm{C} .{ }^{1} \mathrm{H}$ NMR $\left(\mathrm{CDCl}_{3}, 300 \mathrm{MHz}\right): \delta 3.85(3 \mathrm{H}, \mathrm{s}, \mathrm{OMe}), 3.92$ (3H, s, OMe), $6.25\left(2 \mathrm{H}\right.$, broad s, $\left.\mathrm{NH}_{2}\right), 6.92\left(2 \mathrm{H}, \mathrm{d}, \mathrm{J}=8.4 \mathrm{~Hz}, 3\right.$ ' and 5'-H), $7.52\left(2 \mathrm{H}, \mathrm{d}, \mathrm{J}=8,4 \mathrm{~Hz}, 2^{\prime}\right.$ and 6'-H), $8.16(1 \mathrm{H}, \mathrm{d}, \mathrm{J}=2 \mathrm{~Hz}, \mathrm{Ar}-\mathrm{H}), 8.71(1 \mathrm{H}, \mathrm{d}, \mathrm{J}=2 \mathrm{~Hz}, \mathrm{Ar}-\mathrm{H}) \mathrm{ppm} .{ }^{13} \mathrm{C} \mathrm{NMR}\left(\mathrm{CDCl}_{3}, 75.4 \mathrm{MHz}\right)$ : $\delta 51.74(\mathrm{OMe}), 55.34(\mathrm{OMe}), 84.90(\mathrm{C}), 94.17$ (C), 100.63 (2-C), 114.16 (3' and 5'-CH), $114.28(\mathrm{C})$, $119.52(\mathrm{C}), 133.25(\mathrm{CH}), 133.29$ (2' and 6'-CH), $133.78(\mathrm{C}), 144.56(\mathrm{C}), 147.27(\mathrm{C}), 148.56(\mathrm{CH})$, $160.20(\mathrm{C}), 165.18(\mathrm{C}=\mathrm{O})$ ppm. MS (EI): m/z (\%) $338\left(\mathrm{M}^{+}, 100\right), 306$ (39). HRMS M ${ }^{+} \mathrm{C}_{18} \mathrm{H}_{14} \mathrm{~N}_{2} \mathrm{O}_{3} \mathrm{~S}$ : caldt. 338.0725; found 338.0724.

4.1.1.5. Methyl 3-amino-6-[2-(2-aminophenyl)ethynyl]thieno[3,2-b]pyridine-2-carboxylate (2e): Yellow solid (122 mg, 70\%), m.p. 168-170 ${ }^{\circ} \mathrm{C} .{ }^{1} \mathrm{H}$ NMR (DMSO- $\left.d_{6}, 400 \mathrm{MHz}\right) \delta 3.82$ (3H, s, OMe), 5.68 $\left(2 \mathrm{H}\right.$, br s, $\left.\mathrm{NH}_{2}\right), 6.52-6.57(1 \mathrm{H}, \mathrm{m}, \mathrm{Ar}-\mathrm{H}), 6.73(1 \mathrm{H}$, br d, $J=9.2 \mathrm{~Hz}, \mathrm{Ar}-\mathrm{H}), 6.93$ (2H, br s, 2'- $\left.\mathrm{NH}_{2}\right)$, 7.08-7.14 $(1 \mathrm{H}, \mathrm{m}, \mathrm{Ar}-\mathrm{H}), 7.26(1 \mathrm{H}, \mathrm{dd}, J=7.6$ and $1.6 \mathrm{~Hz}, \mathrm{Ar}-\mathrm{H}), 8.63(1 \mathrm{H}, \mathrm{d}, J=1.6 \mathrm{~Hz}, \mathrm{Ar}-\mathrm{H}), 8.85$ $(1 \mathrm{H}, \mathrm{d}, J=1.6 \mathrm{~Hz}, \mathrm{Ar}-\mathrm{H}) \mathrm{ppm} .{ }^{13} \mathrm{C}$ NMR (DMSO-d, $\left.100.6 \mathrm{MHz}\right) \delta 51.57(\mathrm{OMe}), 90.88(\mathrm{C}), 91.60(\mathrm{C})$, $97.94(2-\mathrm{C}), 104.38(\mathrm{C}), 114.03(\mathrm{CH}), 115.69(\mathrm{CH}), 119.02(\mathrm{C}), 130.46(\mathrm{CH}), 131.89(\mathrm{CH}), 132.97(\mathrm{C})$, $133.50(\mathrm{CH}), 144.57(\mathrm{C}), 147.77(\mathrm{C}), 148.51(\mathrm{CH}), 150.15(\mathrm{C}), 164.40(\mathrm{C}=\mathrm{O}) \mathrm{ppm}$. Elemental Anal. calcd for $\mathrm{C}_{17} \mathrm{H}_{13} \mathrm{~N}_{3} \mathrm{O}_{2} \mathrm{~S} \mathrm{C}, 63.14 ; \mathrm{H}, 4.05 ; \mathrm{N}, 12.99 ; \mathrm{S}, 9.92 \%$. Found $\mathrm{C}, 63.05 ; \mathrm{H}, 4.05 ; \mathrm{N}, 12.59 ; \mathrm{S}$, $9.48 \%$.

4.1.1.6. Methyl 3-amino-6-[2-(3-aminophenyl)ethynyl]thieno[3,2-b]pyridine-2-carboxylate (2f): Yellow solid (105 mg, 60\%), m.p. 203-205 ${ }^{\circ} \mathrm{C} .{ }^{1} \mathrm{H}$ NMR (DMSO- $\left.d_{6}, 400 \mathrm{MHz}\right) \delta 3.82$ (3H, s, OMe), 5.30 $\left(2 \mathrm{H}\right.$, br s, $\left.\mathrm{NH}_{2}\right), 6.64(1 \mathrm{H}$, ddd, $\mathrm{J}=8.12 .4$ and $1.2 \mathrm{~Hz}, \mathrm{Ar}-\mathrm{H}), 6.73(1 \mathrm{H}, \mathrm{dt}, \mathrm{J}=7.6$ and $1.2 \mathrm{~Hz}, \mathrm{Ar}-\mathrm{H})$, $6.77(1 \mathrm{H}, \mathrm{m}, \mathrm{Ar}-\mathrm{H}), 6.92\left(2 \mathrm{H}\right.$, br s, $\left.\mathrm{NH}_{2}\right), 7.01(1 \mathrm{H}$, app. t, $J=8 \mathrm{~Hz}, 5$ '-H), $8.57(1 \mathrm{H}, \mathrm{d}, J=2 \mathrm{~Hz}, \mathrm{Ar}-\mathrm{H})$, $8.76(1 \mathrm{H}, \mathrm{d}, J=2 \mathrm{~Hz}, \mathrm{Ar}-\mathrm{H}) \mathrm{ppm} .{ }^{13} \mathrm{C}$ NMR (DMSO-d $\left.6,100.6 \mathrm{MHz}\right) \delta 51.62(\mathrm{OMe}), 84.97(\mathrm{C}), 94.46$ (C), 98.35 (2'-C), 115.17 (4'-CH), 116.14 (2'-CH), 118.42 (C), 118.94 (6'-CH), 121.68 (C), 129.32 (5'$\mathrm{CH}), 133.10(\mathrm{C}), 133.92(\mathrm{CH}), 144.94(\mathrm{C}), 147.65(\mathrm{C}), 148.53(\mathrm{CH}), 148.91(\mathrm{C}), 164.40(\mathrm{C}=\mathrm{O}) \mathrm{ppm}$. MS (EI) $\mathrm{m} / \mathrm{z} 323\left(\mathrm{M}^{+}, 100\right), 291$ (56). HRMS calcd. for $\mathrm{C}_{17} \mathrm{H}_{13} \mathrm{~N}_{3} \mathrm{O}_{2} \mathrm{~S} \mathrm{M}^{+}$323.0728; found 323.0729.

4.1.1.7. Methyl 3-amino-6-[2-(4-aminophenyl)ethynyl]thieno[3,2-b]pyridine-2-carboxylate (2g): Yellow solid (130 mg, 75\%), m.p. $211-213^{\circ} \mathrm{C}$. ${ }^{1} \mathrm{H}$ NMR (DMSO-d, $\left.300 \mathrm{MHz}\right) \delta 3.81(3 \mathrm{H}, \mathrm{s}, \mathrm{OMe}), 5.70$ $\left(2 \mathrm{H}\right.$, br s, $\left.\mathrm{NH}_{2}\right), 6.57\left(2 \mathrm{H}, \mathrm{d}, J=8.4 \mathrm{~Hz}, 3^{\prime}\right.$ and 5' $\left.-\mathrm{CH}\right), 6.91\left(2 \mathrm{H}\right.$, br s, $\left.\mathrm{NH}_{2}\right), 7.25\left(2 \mathrm{H}, \mathrm{d}, J=8.4 \mathrm{~Hz}, 2^{\prime}\right.$ and 6'-CH), $8.46(1 \mathrm{H}, \mathrm{d}, J=2 \mathrm{~Hz}, \mathrm{Ar}-\mathrm{H}), 8.70(1 \mathrm{H}, \mathrm{d}, J=2 \mathrm{~Hz}, \mathrm{Ar}-\mathrm{H}) \mathrm{ppm} .{ }^{13} \mathrm{C}$ NMR (DMSO- $d_{6}, 75.4$ $\mathrm{MHz}) \delta 51.60$ (OMe), 83.87 (C), 96.06 (C), 97.85 (2-C), 107.07 (C), 113.62 (3' and 5'-CH), 119.43 (C), 132.92 (2' and 6'-CH), $132.97(\mathrm{CH}), 133.22(\mathrm{C}), 144.33(\mathrm{C}), 147.77(\mathrm{C}), 148.29(\mathrm{CH}), 150.15(\mathrm{C})$, $164.46(\mathrm{C}=\mathrm{O}) \mathrm{ppm}$. MS (EI) m/z $323\left(\mathrm{M}^{+}, 100\right), 291$ (51). HRMS calcd. for $\mathrm{C}_{17} \mathrm{H}_{13} \mathrm{~N}_{3} \mathrm{O}_{2} \mathrm{~S} \mathrm{M}^{+} 323.0728$; found 323.0727 .

4.1.1.8. Methyl 3-amino-6-\{2-[4-(dimethylamino)phenyl]ethynyl $\}$ thieno[3,2-b]pyridine-2carboxylate (2h): Green solid (152 mg, 80\%), m.p. 191-193 ${ }^{\circ} \mathrm{C} .{ }^{1} \mathrm{H}$ NMR (DMSO- $\left.d_{6}, 300 \mathrm{MHz}\right) \delta 2.96$ $(6 \mathrm{H}, \mathrm{s}, 2 \times \mathrm{Me}), 3.81(3 \mathrm{H}, \mathrm{s}, \mathrm{OMe}), 6.73\left(2 \mathrm{H}, \mathrm{d}, J=8.7 \mathrm{~Hz}, 3^{\prime}\right.$ and 5' $\left.-\mathrm{CH}\right), 6.92\left(2 \mathrm{H}, \mathrm{br} \mathrm{s}, \mathrm{NH}_{2}\right), 7.40(2 \mathrm{H}$, $\mathrm{d}, J=8.7 \mathrm{~Hz}, 2$ ' and 6'-CH), $8.49(1 \mathrm{H}, \mathrm{d}, J=2 \mathrm{~Hz}, \mathrm{Ar}-\mathrm{H}), 8.72(1 \mathrm{H}, \mathrm{d}, \mathrm{J}=2 \mathrm{~Hz}, \mathrm{Ar}-\mathrm{H}) \mathrm{ppm} .{ }^{13} \mathrm{C}$ NMR $\left(\mathrm{DMSO}-d_{6}, 75.4 \mathrm{MHz}\right) \delta 39.59(2 \times \mathrm{Me}), 51.56(\mathrm{OMe}), 84.51$ (C), $95.60(\mathrm{C}), 97.86(2-\mathrm{C}), 107.33$ (1'C), 111.79 (3' and 5'-CH), $119.24(\mathrm{C}), 132.66$ (2' and 6'-CH), $133.05(\mathrm{CH}), 133.16(\mathrm{C}), 144.36(3 \mathrm{a}-\mathrm{C})$, $147.72(\mathrm{C}), 148.28(\mathrm{CH}), 150.44$ (4'-C), $164.41(\mathrm{C}=\mathrm{O}) \mathrm{ppm} . \mathrm{MS}(\mathrm{EI}) \mathrm{m} / \mathrm{z} 351\left(\mathrm{M}^{+}, 100\right), 319$ (33). HRMS calcd. for $\mathrm{C}_{19} \mathrm{H}_{17} \mathrm{~N}_{3} \mathrm{O}_{2} \mathrm{~S} \mathrm{M}^{+} 351.1041$; found 351.1042. 
4.1.1.9. Methyl 3-amino-6-[2-(2-fluorophenyl)ethynyl]thieno[3,2-b]pyridine-2-carboxylate (2i): Yellow solid (132 mg, 75\%), m.p. $174-176{ }^{\circ} \mathrm{C} .{ }^{1} \mathrm{H} \mathrm{NMR}\left(\mathrm{CDCl}_{3}, 400 \mathrm{MHz}\right) \delta 3.93(3 \mathrm{H}, \mathrm{s}, \mathrm{OMe}), 6.22$ $\left(2 \mathrm{H}\right.$, br s, $\left.\mathrm{NH}_{2}\right), 7.14-7.18(2 \mathrm{H}, \mathrm{m}, \mathrm{Ar}-\mathrm{H}), 7.35-7.41(1 \mathrm{H}, \mathrm{m}, \mathrm{Ar}-\mathrm{H}), 7.53-7.58(1 \mathrm{H}, \mathrm{m}, \mathrm{Ar}-\mathrm{H}), 8.21(1 \mathrm{H}, \mathrm{d}$, $J=2 \mathrm{~Hz}, \mathrm{Ar}-\mathrm{H}), 8.75(1 \mathrm{H}, \mathrm{d}, J=2 \mathrm{~Hz}, \mathrm{Ar}-\mathrm{H}) \mathrm{ppm} .{ }^{13} \mathrm{C} \mathrm{NMR}\left(\mathrm{CDCl}_{3}, 100.6 \mathrm{MHz}\right) \delta 51.75(\mathrm{OMe}), 87.10$ (C), 91.03 (C), 101.00 (2-C), 111.06 (d, J=16 Hz, C), 115.68 (d, J = 21 Hz, CH), 118.68 (C), 124,12 (d, $J=3 \mathrm{~Hz}, \mathrm{CH}), 130.78$ (d, $J=8 \mathrm{~Hz}, \mathrm{CH}), 133.53(\mathrm{~d}, J=13 \mathrm{~Hz}, \mathrm{CH}), 133.63(\mathrm{CH}), 145.29(\mathrm{C}), 147.31$ (C), $148.77(\mathrm{CH}), 162.73(\mathrm{~d}, J=251 \mathrm{~Hz}, \mathrm{C}-\mathrm{F}), 165.16(\mathrm{C}=\mathrm{O}) \mathrm{ppm}$. Elemental Analysis calcd. for $\mathrm{C}_{17} \mathrm{H}_{11} \mathrm{FN}_{2} \mathrm{O}_{2} \mathrm{~S} \mathrm{C}, 62.57 ; \mathrm{H}, 3.40 ; \mathrm{N}, 8.58 ; \mathrm{S}, 9.83 \%$. Found C, 62.39; H, 3.43; N, 8.40; S, 9.43\%.

4.1.1.10. Methyl 3-amino-6-[2-(4-fluorophenyl)ethynyl]thieno[3,2-b]pyridine-2-carboxylate (2j): Yellow solid (159 mg, 90\%) m.p. 167-169 ${ }^{\circ} \mathrm{C} .{ }^{1} \mathrm{H}$ NMR $\left(\mathrm{CDCl}_{3}, 400 \mathrm{MHz}\right) \delta 3.93(3 \mathrm{H}, \mathrm{s}, \mathrm{OMe}), 6.23$ $\left(2 \mathrm{H}\right.$, br s, $\left.\mathrm{NH}_{2}\right), 7.07-7.11(2 \mathrm{H}, \mathrm{m}, \mathrm{Ar}-\mathrm{H}), 7.54-7.58(2 \mathrm{H}, \mathrm{m}, \mathrm{Ar}-\mathrm{H}), 8.17(1 \mathrm{H}, \mathrm{d}, J=2 \mathrm{~Hz}, \mathrm{Ar}-\mathrm{H}), 8.71$ $(1 \mathrm{H}, \mathrm{d}, J=2 \mathrm{~Hz}, \mathrm{Ar}-\mathrm{H}) \mathrm{ppm} .{ }^{13} \mathrm{C} \mathrm{NMR}\left(\mathrm{CDCl}_{3}, 100.6 \mathrm{MHz}\right) \delta 51.76(\mathrm{OMe}), 85.83(\mathrm{C}), 92.75(\mathrm{C})$, 100.89 (2-C), 115.88 (d, $J=21 \mathrm{~Hz}, 3$ ' and 5'-CH), 118.43 (d, $J=3 \mathrm{~Hz}, \mathrm{C}), 118.92(\mathrm{C}), 133.42(\mathrm{CH})$, $133.70\left(\mathrm{~d}, J=10 \mathrm{~Hz}, 2^{\prime}\right.$ and 6'-CH ), $133.76(\mathrm{C}), 145.07(\mathrm{C}), 147.29(\mathrm{C}), 148.69(\mathrm{CH}), 162.92(\mathrm{~d}, J=$ $252 \mathrm{~Hz}, \mathrm{C}-\mathrm{F}), 165.17(\mathrm{C}=\mathrm{O}) \mathrm{ppm}$. Elemental Analysis calcd. for $\mathrm{C}_{17} \mathrm{H}_{11} \mathrm{FN}_{2} \mathrm{O}_{2} \mathrm{~S} \mathrm{C}, 62.57 ; \mathrm{H}, 3.40 ; \mathrm{N}$, $8.58 ; \mathrm{S}, 9.83 \%$. Found C, 62.25; H, 3.53; N, 8.24; S, 9.99\%.

4.1.1.11. Methyl 3-amino-6-[2-(pyridin-2-yl)ethynyl]thieno[3,2-b]pyridine-2-carboxylate (2k): Yellow solid (117 mg, 70\%), m.p. 226-228 ${ }^{\circ} \mathrm{C} .{ }^{1} \mathrm{H}$ NMR (DMSO-d $\left.6, \mathrm{~T}=60^{\circ} \mathrm{C}, 300 \mathrm{MHz}\right) \delta 3.84(3 \mathrm{H}$, s, $\mathrm{OMe}), 6.81\left(2 \mathrm{H}\right.$, br s, $\left.\mathrm{NH}_{2}\right), 7.42-7.47(1 \mathrm{H}, \mathrm{m}, \mathrm{Ar}-\mathrm{H}), 7.70(1 \mathrm{H}, \mathrm{d}, J=7.8 \mathrm{~Hz}, \mathrm{Ar}-\mathrm{H}), 7.85-7.91(1 \mathrm{H}, \mathrm{m}$, Ar-H), 8.63-8.66 (2H m, Ar-H), $8.84(1 \mathrm{H}, \mathrm{d}, J=2 \mathrm{~Hz}, \mathrm{Ar}-\mathrm{H}) \mathrm{ppm} .{ }^{13} \mathrm{C}$ NMR (DMSO- $d_{6}, \mathrm{~T}=60{ }^{\circ} \mathrm{C}, 75.4$ $\mathrm{MHz}) \delta 51.33$ (OMe), $85.03(\mathrm{C}), 92.47$ (C), 99.06 (2-C), $117.04(\mathrm{C}), 123.66(\mathrm{CH}), 127.33(\mathrm{CH}), 132.83$ (C), $134.32(\mathrm{CH}), 136.54(\mathrm{CH}), 141.52(\mathrm{C}), 145.33(\mathrm{C}), 147.22(\mathrm{C}), 148.43(\mathrm{C}), 150.01(\mathrm{CH}), 164.08$ $(\mathrm{C}=\mathrm{O}) \mathrm{ppm}$. MS (EI) m/z $309\left(\mathrm{M}^{+}, 100\right), 277$ (63). HRMS calcd. for $\mathrm{C}_{16} \mathrm{H}_{11} \mathrm{~N}_{3} \mathrm{O}_{2} \mathrm{~S} \mathrm{M}^{+} 309.0572$; found 309.0571 .

4.1.1.12. Methyl 3-amino-6-[2-(pyridin-3-yl)ethynyl]thieno[3,2-b]pyridine-2-carboxylate (2l): Yellow solid (150 mg, 90\%), m.p. 205-207 ${ }^{\circ} \mathrm{C}$. ${ }^{1} \mathrm{H}$ NMR $\left(\mathrm{CDCl}_{3}, 400 \mathrm{MHz}\right) \delta 3.93(3 \mathrm{H}, \mathrm{s}, \mathrm{OMe}), 6.22$ $\left(2 \mathrm{H}\right.$, br s, $\left.\mathrm{NH}_{2}\right), 7.36-7.38(1 \mathrm{H}, \mathrm{m}, \mathrm{Ar}-\mathrm{H}), 7.44-7.50(1 \mathrm{H}, \mathrm{m}, \mathrm{Ar}-\mathrm{H}), 7.64-7.70(1 \mathrm{H}, \mathrm{m}, \mathrm{Ar}-\mathrm{H}), 7.87(1 \mathrm{H}, \mathrm{d}$, $J=8 \mathrm{~Hz}, \mathrm{Ar}-\mathrm{H}), 8.21(1 \mathrm{H}, \mathrm{d}, J=2 \mathrm{~Hz}, \mathrm{Ar}-\mathrm{H}), 8.74(1 \mathrm{H}, \mathrm{d}, J=2 \mathrm{~Hz}, \mathrm{Ar}-\mathrm{H}) \mathrm{ppm} .{ }^{13} \mathrm{C} \mathrm{NMR}\left(\mathrm{CDCl}_{3}, 100.6\right.$ $\mathrm{MHz}): \delta 51.78(\mathrm{OMe}), 89.52(\mathrm{C}), 90.08(\mathrm{C}), 101.18(2-\mathrm{C}), 118.18(\mathrm{C}), 119.78(\mathrm{C}), 123.29(\mathrm{CH}), 128.55$ (C), $132.02(\mathrm{CH}), 133.61(\mathrm{C}), 133.65(\mathrm{CH}), 138.78(\mathrm{CH}), 145.54(\mathrm{C}), 147.27(\mathrm{C}), 148.74(\mathrm{CH}), 165.13$ $(\mathrm{C}=\mathrm{O})$ ppm. MS (EI) m/z $309\left(\mathrm{M}^{+}, 53\right), 277$ (100). HRMS calcd. for $\mathrm{C}_{16} \mathrm{H}_{11} \mathrm{~N}_{3} \mathrm{O}_{2} \mathrm{~S} \mathrm{M}^{+} 309.0572$; found 309.0578 .

4.1.1.13. Methyl 3-amino-6-[2-(thien-3-yl)ethynyl)]thieno[3,2-b]pyridine-2-carboxylate (2m): Yellow solid (119 mg, 70\%), m.p. 165-167 ${ }^{\circ} \mathrm{C} .{ }^{1} \mathrm{H}$ NMR $\left(\mathrm{CDCl}_{3}, 400 \mathrm{MHz}\right) \delta 3.93(3 \mathrm{H}, \mathrm{s}, \mathrm{OMe}), 6.22(2 \mathrm{H}, \mathrm{br} \mathrm{s}$, $\left.\mathrm{NH}_{2}\right), 7.24(1 \mathrm{H}, \mathrm{dd}, J=4.8$ and $1.2 \mathrm{~Hz}, \mathrm{Ar}-\mathrm{H}), 7.35(1 \mathrm{H}, \mathrm{dd}, J=4.8$ and $2.8 \mathrm{~Hz}, \mathrm{Ar}-\mathrm{H}), 7.62(1 \mathrm{H}, \mathrm{dd}, J$ $=2.8$ and $1.2 \mathrm{~Hz}, \mathrm{Ar}-\mathrm{H}), 8.16(1 \mathrm{H}, \mathrm{d}, J=2 \mathrm{~Hz}, \mathrm{Ar}-\mathrm{H}), 8.71(1 \mathrm{H}, \mathrm{d}, J=2 \mathrm{~Hz}, \mathrm{Ar}-\mathrm{H}) \mathrm{ppm} .{ }^{13} \mathrm{C}$ NMR $\left(\mathrm{CDCl}_{3}, 75.4 \mathrm{MHz}\right) \delta 51.74(\mathrm{OMe}), 85.72(\mathrm{C}), 89.05$ (C), 100.78 (2-C), $119.09(\mathrm{C}), 121.40(\mathrm{C}), 125.76$ $(\mathrm{CH}), 129.74(2 \times \mathrm{CH}), 133.32(\mathrm{CH}), 133.70(\mathrm{C}), 144.97(\mathrm{C}), 147.32(\mathrm{C}), 148.68(\mathrm{CH}), 165.18(\mathrm{C}=\mathrm{O})$ ppm. MS (EI) m/z $314\left(\mathrm{M}^{+}\right.$, 100), 282 (90). HRMS calcd. for $\mathrm{C}_{15} \mathrm{H}_{10} \mathrm{~N}_{2} \mathrm{O}_{2} \mathrm{~S}_{2} \mathrm{M}^{+} 314.0184$; found 314.0183 .

\subsection{Biological activity}

\subsubsection{Material and Methods}

\subsubsection{Reagents}

Fetal bovine serum (FBS), L-glutamine, phosphate buffered saline (PBS) and trypsin were from Gibco Invitrogen Co. (Scotland, UK). RPMI-1640 medium was from Cambrex (New Jersey, USA). Acetic acid, dimethyl sulfoxide (DMSO), doxorubicin, penicillin, streptomycin, ethylenediaminetetraacetic acid (EDTA), sulforhodamine B (SRB) and trypan blue were from SigmaChemical Co. (Saint Louis, USA). Tricloroacetic acid (TCA) and Tris were sourced from Merck (Darmstadt, Germany). 


\subsubsection{Solutions of the compounds}

Stock solutions of the tested compounds were prepared in DMSO and kept at $-70^{\circ} \mathrm{C}$. Appropriate dilutions were freshly prepared in the test medium just prior to the assays. The effect of the vehicle solvent (DMSO) on the growth of the cell lines was evaluated by exposing untreated control cells to the maximum concentration of DMSO used in the assays $(0.25 \%)$. No influence was found (data not shown).

\subsubsection{Cell cultures}

Three human tumor cell lines, MCF-7 (breast adenocarcinoma), NCI-H460 (non-small cell lung cancer) and A375-C5 (melanoma) were used. MCF-7 and A375-C5 were obtained from the European Collection of Cell Cultures (ECACC, Salisbury, UK), and NCI-H460 was kindly provided by the National Cancer Institute (NCI, Bethesda, USA). They were routinely maintained as adherent cell cultures in RPMI-1640 medium supplemented with 5\% heat-inactivated FBS, $2 \mathrm{mM}$ glutamine and antibiotics (penicillin 100 $\mathrm{U} / \mathrm{mL}$, streptomycin $100 \mu \mathrm{g} / \mathrm{mL}$ ), at $37^{\circ} \mathrm{C}$ in a humidified atmosphere containing $5 \% \mathrm{CO}_{2}$. Exponentially growing cells were obtained by plating $1.5 \times 10^{5} \mathrm{cells} / \mathrm{mL}$ for MCF-7 and $0.75 \times 10^{5} \mathrm{cells} / \mathrm{mL}$ for A375-C5 and NCI-H460, followed by a 24-h incubation.

\subsubsection{Growth inhibition assay}

The effects on the in vitro growth of human tumor cell lines were evaluated according to the procedure adopted by the NCI (USA) in their "In vitro Anticancer Drug Discovery Screen", using the proteinbinding dye sulforhodamine B to assess cell growth.[8,9] Briefly, exponentially growing cells were exposed for $48 \mathrm{~h}$, in 96-well microtiter plates, to five serial dilutions of each test compound, starting from a maximum concentration of $150 \mu \mathrm{M}$ (if possible). Following the exposure period adherent cells were fixed in situ with TCA, washed and stained with SRB. The bound stain was solubilized and the absorbance was measured at $492 \mathrm{~nm}$ in a plate reader (Bio-tek Instruments Inc., Powerwave XS, Wincoski, USA). Dose-response curves were obtained for each test compound and cell line, and the growth inhibition of $50 \%\left(\mathrm{GI}_{50}\right)$, corresponding to the concentration of the compounds that inhibited $50 \%$ of the net cell growth was calculated as described elsewhere.[9] Doxorubicin was tested in the same manner, to be used as a positive control.

\subsubsection{Flow cytometric assays for cell cycle distribution analysys and apoptosis detection with annexin V-FITC/PI assay}

NCI-H460 cells were plated at $0.75 \times 10^{5} \mathrm{cells} / \mathrm{mL}$ in 6 -well plates and left incubating for $24 \mathrm{~h}$. Cells were then incubated with complete medium only (control), medium with the compound's solvent (DMSO) or with compounds $\mathbf{2 d}, \mathbf{2 e}$ and $\mathbf{2} \mathbf{g}$ at their respective $\mathrm{GI}_{50}$ concentrations previously obtained by the SRB assay $(0.32,5.7$ and $8.9 \mu \mathrm{M})$. Cells were harvested following a $48 \mathrm{~h}$ incubation with the compounds and further processed for either cell cycle analysis or apoptosis detection. For cell cycle analysis, cells were fixed in $70 \%$ ethanol and subsequently resuspended in PBS containing $0.1 \mathrm{mg} / \mathrm{mL}$ RNase A and $5 \mu \mathrm{g} / \mathrm{mL}$ propidium iodide, prior to analysis. Induced apoptosis was assayed by the Human Annexin V-FITC/PI apoptosis Kit (Bender MedSystems, Vienna, Austria) according to the manufacturer's instructions.[10] Cellular DNA content (for cell cycle distribution analysis and presence of sub-G1 peak, suggestive of apoptosis induction) and measurement of phosphatidylserine externalization were analyzed using an Epics XL-MCL Coulter flow cytometer plotting at least 20,000 events per sample. Cell cycle distribution and apoptosis data analysis was subsequently performed using FlowJo 7.2 software (Tree Star, Inc.). Experiments were performed in duplicate in three to six independent occasions and the average and standard error of the obtained results were presented. Statistical analysis was performed for the induction of apoptosis results by the non-parametric Friedman's test followed by Dunn's Post-test using GraphPad Prism 5 software. $P$ values $<0.05$ were considered as statistically significant.

\section{Acknowledgments}

To the Foundation for Science and Technology (FCT, Portugal) through the financial support to the research centres, the national NMR network (Bruker Avance III 400) REDE/1517/RMN/2005, the research project PTDC/QUI-QUI/111060/2009 which is also financed by COMPETE/QREN/EU. RCC and LAVS acknowledge FCT for their PhD (SFRH/BD/29274/2006) and post-doctoral (SFRH/BPD/29112/2006) grants, respectively. GMA is supported by FCT and the European Social Fund. IPATIMUP is an Associate Laboratory of the Portuguese Ministry of Science, Technology and Higher Education and is partially supported by FCT. 


\section{References}

[1] M. J. Munchhof, J. S. Beebe, J. M. Casavant, B. A. Cooper, J. L. Doty, R. C. Hidgon, S. M. Hillerman, C. I. Doderstrom, E. A. Knauth, M. A. Marx, A. M. K. Rossi, S. B. Sobolov, J. Sun, Bioorg. Med. Chem. Lett. 14 (2004) 21-24.

[2] S. Claridge, F. Raeppel, M.-C. Granger, N. Bernstein, O. Saavedra, L. Zhan, D. Llewellyn, A. Wahhab, R. Deziel, J. Rahil, N. Beaulieu, H. Nguyen, I. Dupont, A. Barsalou, C. Beaulieu, I. Chute, S. Gravel, M.-F. Robert, S. Lefebvre, M. Dubay, R. Pascal, J. Gillespie, Z. Jin, J. Wang, J.M. Besterman, A. R. MacLeod; A. Vaisburg, Bioorg. Med. Chem. Lett. 18 (2008) 2793-2798.

[3] S. Raeppel, S. Claridge, O. Saavedra, F. Gaudette, L. Zhan, M. Mannion, N. Zhou, F. Raeppel, M.-C. Granger, L. Isakovick, R. Deziel, H. Nguyen, N. Beaulieu, C. Beaulieu, I. Dupont, M.-F. Robert, S. Lefebvre, M. Dubay, J. Rahil, J. Wang, H. Ste-Croix, A. R. MacLeod, J.Besterman, A. Vaisburg, Bioorg. Med. Chem. Lett. 19 (2009) 1323-1328.

[4] D. H. Boschelli, B. Wu, A. C. B. Susa, H. Durutlic, F. Ye, Y. Raifeld, J. M. Golas, F. Boschelli, J. Med. Chem. 47 (2004) 6666-6668.

[5] R. C. Calhelha, M.-J. R. P. Queiroz, Tetrahedron Lett. 51 (2010) 281-283.

[6] For a recent review on the Sonogashira reaction see: R. Chincilla, C. Nájera Chem. Rev. 107 (2007) 874-922.

[7] M.-J. R. P. Queiroz, R. C. Calhelha, L. A. Vale-Silva, E. Pinto, M. S.-J. Nascimento, Eur. J. Med. Chem. 44 (2009) 1893-1899.

[8] P. Skehan, R. Storeng, D. Scudiero, A. Monks, J. McMahon, D. Vistica, J. T. Warren, H. Bokesch, S. Kenney, M. R. Boyd, J. Natl. Cancer Inst. 83 (1991) 757-776.

[9] A. Monks, D. Scudiero, P. Skehan, R. Shoemaker, K. Paull, D. Vistica, C. Hose, J. Langley, P. Cronise, A. Vaigro-Wolff, M. Gray-Goodrich, H. Campbell, J. Mayo, M. Boyd, J. Natl. Cancer Inst. 83 (1991) 757-776.

[10] G. M. Almeida, T. L. Duarte, P. B. Farmer, W. P. Steward, G. D. D. Jones, Int. J. Cancer 122 (2008) 1810-1819. 